\title{
NEW MECHANISMS FOR THE POWER SWITCHER WITH V EXTINGUISHING CHAMBERS
}

\author{
OVIDIU ANTONESCU ${ }^{1}$, NATAȘA NEDELA ${ }^{2}$, PĂUN ANTONESCU ${ }^{1 *}$ \\ 1 "Politehnica" University of Bucharest, Splaiul Independenţei 313, sector 6, București, \\ 060042, Romania \\ 2 "Enel Energie Muntenia” S.A., Romania
}

\begin{abstract}
Starting from an existing V power switcher mechanism, the paper presents two new kinematic schemes of the $\mathrm{V}$ switcher mechanisms for medium and high power. The proposed schemes consist of triadic chains that ensure transmission angles near to the optimum values. The geometric and kinematic analyses of the proposed mechanisms, with। driven components that have to move with high speeds on disengaging process in the extinguishing chamber, have been achieved. In the second section of the paper, an optimum kinematic scheme of the $\mathrm{V}$ power switcher with triadic chain type $4 \mathrm{R}+2 \mathrm{~T}$ has been presented.
\end{abstract}

Keywords: power switcher mechanism, structural synthesis, kinematic scheme, triadic chain

\section{INTRODUCTION}

We consider the constructive scheme of a power switch (Figure 1) for $110 \mathrm{kV}$ and $1600 \mathrm{~A}$ [1] with two put-out chambers mounted in $\mathrm{V}[2,3,4]$. These power switched are mounted within outdoor electrical systems (Figure 1.a), allowing for multiple interruptions of the electric arc by means of two or four serial put-out chambers $[3,4]$.

The drive by means of a single actuator of the two mechanisms of the type equalizing bar - guide allows for the simultaneous displacement of the translational bars of the mobile contacts in the put-out chambers. This mechanism breaks the electric circuit in two serial places.

The insulating column 1 (Figure 1.a) enables insulation from the ground of the two put-out chambers 3 in $\mathrm{V}$, as well as the insulation of the under-pressure part of the switcher 2 .

Each pole of the switcher is driven by means of an oleo-pneumatic actuator [4], and for a three-pole switch three actuators are necessary.

The main components of the $\mathrm{V}$ switch (Figure 1.b) are: the bar of the mobile contact (1), the equalizing bar of the mechanism (2), the double effect hydraulic device (3), the mechanism casing (4), the locking device (5), the double guide bars (6), the oil gauge in the mechanism casing (7), the put-out chamber (8), the insulating cylinder (9), the upper casing cap (10), the lower cap (11), the fixed top contact (12), the lower fixed contact (13), the porcelain insulator (14), the oil gauge in the upper casing (15).

\footnotetext{
${ }^{*}$ Corresponding author, email: panton38@ hotmail.com
}

(c) 2016 Alma Mater Publishing House 

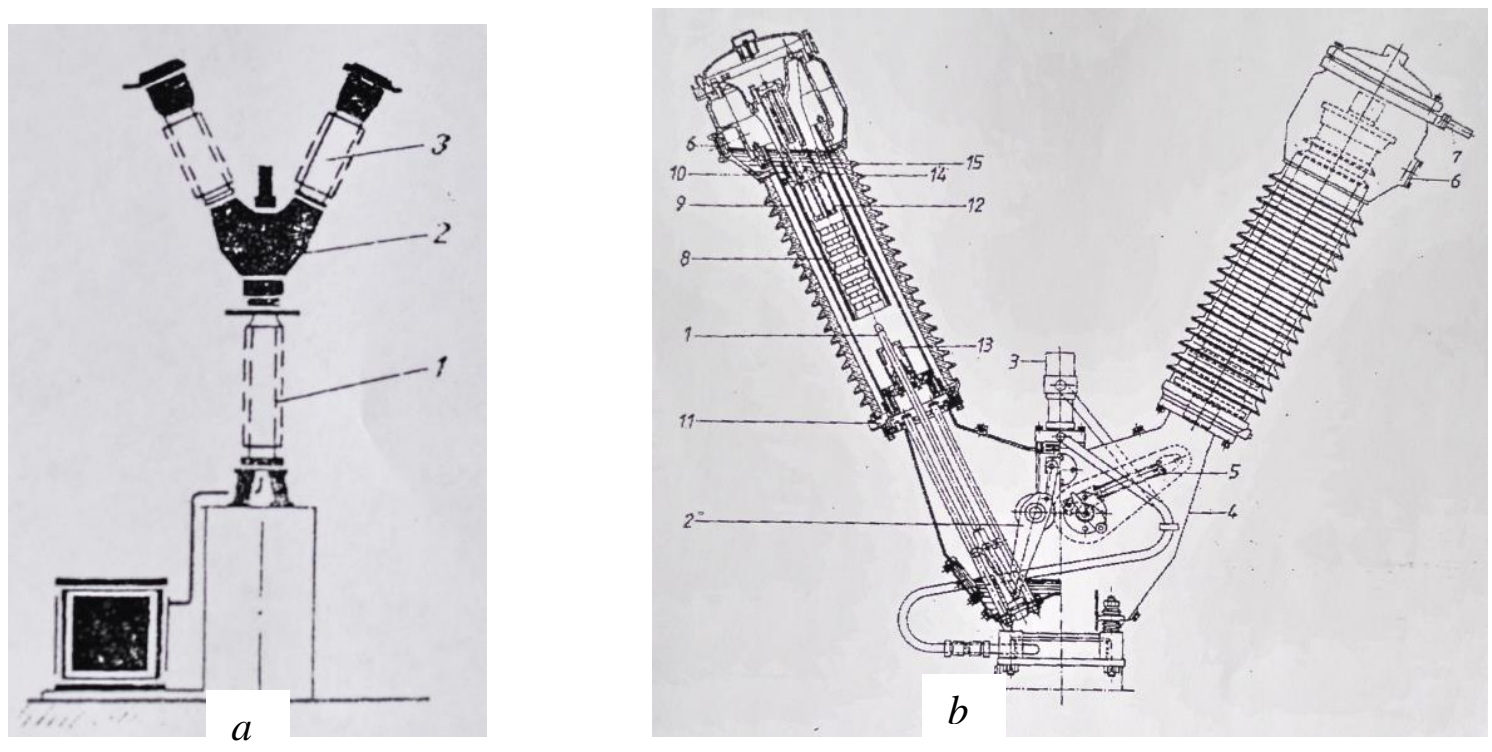

Fig. 1. The constructive scheme of the V power switch of the IO-110-1600 type.

\section{STRUCTURAL SYNTHESIS OF THE TRIADIC CHAIN MECHANISM}

The current mechanism of the power switcher with $\mathrm{V}$ placed extinguishing chambers (Figure 2.a) is based on two kinematic dyadic chains $T+(3 R)+(2 R+T)$. Keeping the same structure of the kinematic couplings used $[2,3]$, the two dyadic chains [1] can be configured into a triadic chain (Figure 2.b).

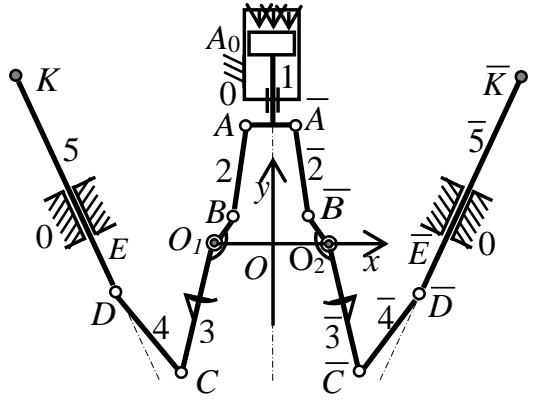

a)

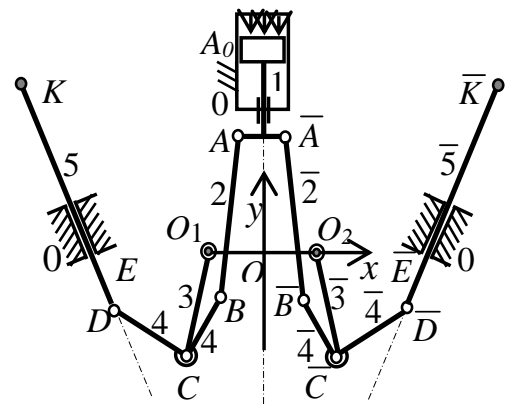

b)

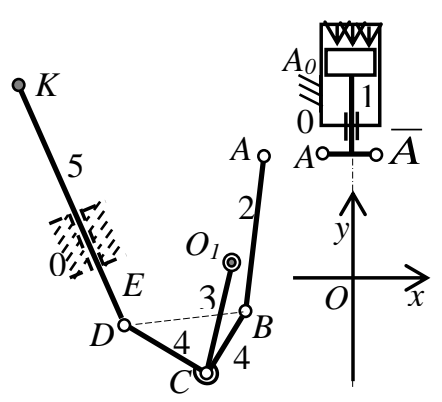

c)

Fig. 2. The kinematic diagram:

a) current; b) new; c) triadic chains LT

With the current kinematic scheme (Figure 2.a), the structural - topological formula of the leading mechanism is [1]:

$$
M M=M A(0,1)+\left\{\begin{array}{l}
L D(2,3)+L D(4,5) \\
L D(\overline{2}, \overline{3})+L D(\overline{4}, \overline{5})
\end{array}\right.
$$

With the new kinematic scheme (fig.2b) the structural - topological formula is written:

$$
M M=M A(0,1)+\left\{\begin{array}{l}
L T(2,3,4,5) \\
L T(\overline{2}, \overline{3}, \overline{4}, \overline{5})
\end{array}\right.
$$

For this new structural and geometrical scheme, the kinematic elements 4 and $\overline{4}$ are central elements of the $3^{\text {rd }}$ degree of the corresponding triadic chain $\operatorname{LT}(2,3,4,5)$ and $L T(\overline{2}, \overline{3}, \overline{4}, \overline{5})$ of the $5 \mathrm{R}+\mathrm{T}$ type (Figure 2.c). 


\section{SYNTHESIS OF A NEW GEOMETRICAL VERSION OF THE V MECHANISM}

We can design structural geometrical versions of the triadic chains $\operatorname{LT}(2,3,4,5)$ and $L T(\overline{2}, \overline{3}, \overline{4}, \overline{5})$, where the 3 articulations (B, C and D) respectively $(\bar{B}, \bar{C}, \bar{D})$ of the central elements 4 and $\overline{4}$ are collinear (Figure 3.a).

We can notice the particularity of this kinematic scheme of the triadic chain LT (Figure 3.a) which consists in placing the three articulations $\mathrm{B}, \mathrm{C}$ and $\mathrm{D}$ on the same straight line. The rotation semi-couplings $\mathrm{A}$ and $\mathrm{C}_{0}$, as well as the translational semi-coupling $\mathrm{E}$ are called [1] potential kinematic couplings by means of which the triadic chain $\mathrm{LT}(2,3,4,5)$ is connected to the leading element 1 and to the fixed element 0 .

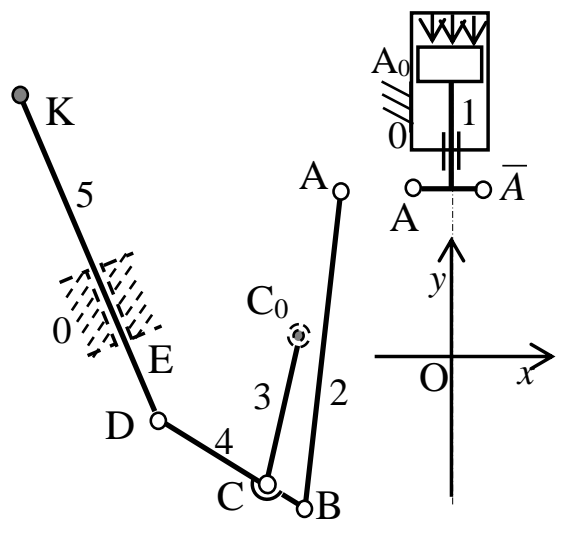

a)

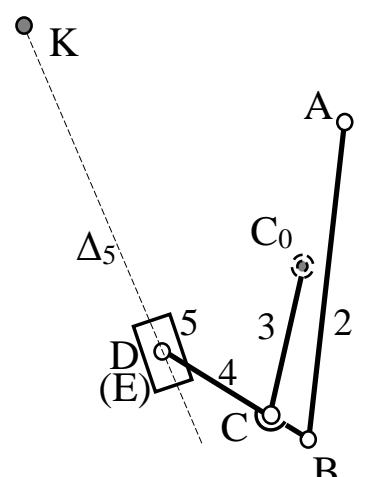

b)

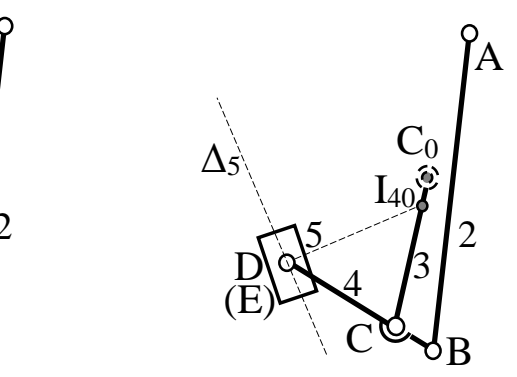

c)

Fig. 3. Version of the kinematic scheme of the triadic chain $\mathrm{LT}(5 \mathrm{R}+\mathrm{T})$.

The equivalent kinematic scheme of the triadic chain $\operatorname{LT}(2,3,4,5)$ is obtained by replacing the bar 5 with a shoe $5^{*}$ that slides along the fixed guide $\Delta_{5}$ (Figure 3.b). With this triadic chain LT $(2,3,4,5)$ we may notice that the central element 4 having a rotation - translational motion (plane-parallel) does an instantaneous rotation around the $I_{40}$ point (the instantaneous rotation centre) situated upon the intersection of the equalizing bar 3 with the line drawn through the articulation $\mathrm{D}$ and perpendicular on the guide $\Delta_{5}$ (Figure 3.c).

\section{KINEMATIC GEOMETRY OF THE TRIADIC CHAIN MECHANISM}

We shall consider the kinematic scheme of the $3^{\text {rd }}$ class mechanism (with a triadic chain) of a $\mathrm{V}$ power switcher (Figure 4.a).

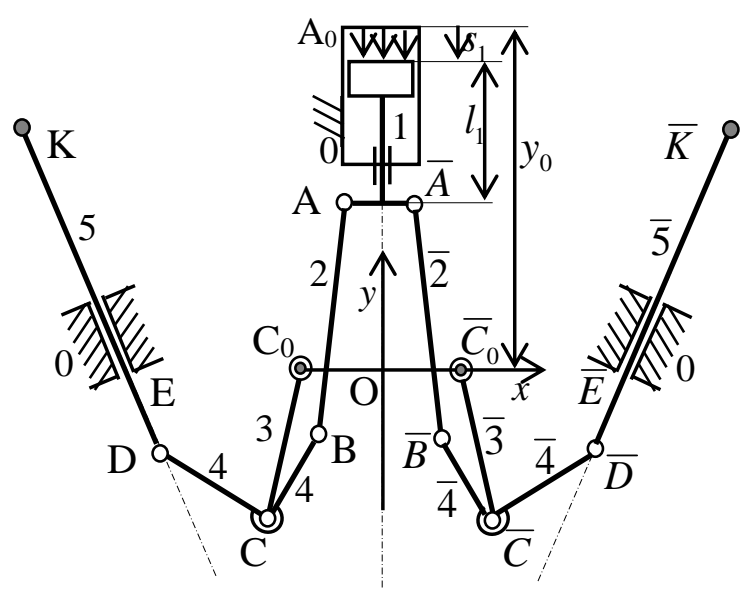

a)

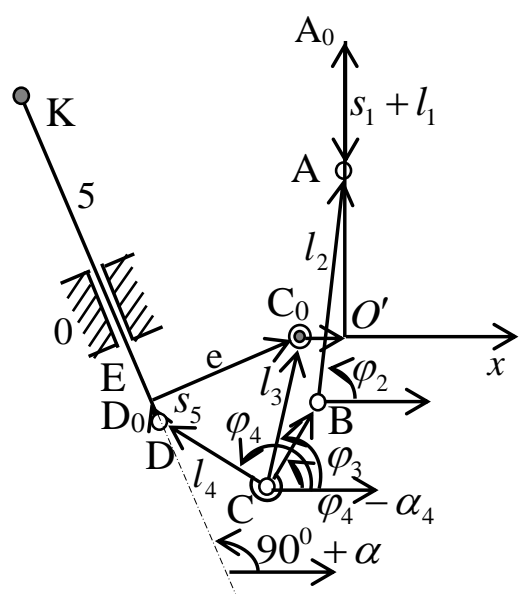

b)

Fig. 4. Kinematic scheme of the proposed mechanism $(a)$ and the associated vector contour lines $(b)$. 
For a geometrical kinematic analysis of the proposed mechanism, we isolate the triadic chain LT $(2,3,4,5)$ of the $5 \mathrm{R}+\mathrm{T}$ type (Figure 4.b). We identify two vector contour lines associated to this kinematic chain, where each side is suitably oriented [1] and positioned by means of the trigonometrically oriented angle.

The two independent contour lines selected are (Figure 4.b): $\mathrm{O}^{\prime} \mathrm{A}_{0} \mathrm{ABCC}_{0} \mathrm{O}^{\prime}$ and $\mathrm{C}_{0} \mathrm{CDD}_{0} \mathrm{C}_{0}$.

For each independent contour line we write the closing vector equation:

$$
\begin{aligned}
& \left(\mathrm{O}^{\prime} \mathrm{A}_{0} \mathrm{ABCC} \mathrm{O}^{\prime}\right): \overrightarrow{O^{\prime} A_{0}}+\overrightarrow{A_{0} A}-\overrightarrow{B A}-\overrightarrow{C B}+\overrightarrow{C C_{0}}+\overrightarrow{C_{0} O^{\prime}}=0 \\
& \left(\mathrm{C}_{0} \mathrm{CDD}_{0} \mathrm{C}_{0}\right): \overrightarrow{C C_{0}}-\overrightarrow{C D}-\overrightarrow{D D_{0}}-\overrightarrow{D_{0} C_{0}}=0
\end{aligned}
$$

The two vector equations are written in a suitable way so as to have the vectors containing unknown variables to the left:

$$
\begin{aligned}
& \overrightarrow{B A}+\overrightarrow{C B}-\overrightarrow{C C_{0}}=\overrightarrow{O^{\prime} A_{0}}+\overrightarrow{A_{0} A}+\overrightarrow{C_{0} O^{\prime}} \\
& \overrightarrow{C C_{0}}-\overrightarrow{C D}-\overrightarrow{D D_{0}}=\overrightarrow{D_{0} C_{0}}
\end{aligned}
$$

The scaling equations related to the vector equations (5) and (6) are expressed as follows (Figure 4.b):

$$
\begin{aligned}
& l_{2} \cos \varphi_{2}+l_{4}^{\prime} \cos \left(\varphi_{4}-\alpha_{4}\right)-l_{3} \cos \varphi_{3}=C_{0} O^{\prime} ; \\
& l_{2} \sin \varphi_{2}+l_{4}^{\prime} \sin \left(\varphi_{4}-\alpha_{4}\right)-l_{3} \sin \varphi_{3}=y_{0}-l_{1}-s_{1} ; \\
& l_{3} \cos \varphi_{3}-l_{4} \cos \varphi_{4}-s_{5} \cos \left(90^{0}+\alpha\right)=e \cdot \cos \alpha ; \\
& l_{3} \sin \varphi_{3}-l_{4} \sin \varphi_{4}-s_{5} \sin \left(90^{0}+\alpha\right)=e \cdot \sin \alpha .
\end{aligned}
$$

The system (7) consists of four scaling equations with four unknown variables, of which three angles $\left(\varphi_{2}, \varphi_{3}, \varphi_{4}\right)$ and a linear displacement $\left(s_{5}\right)$ :

$$
\left\{\begin{array}{c}
l_{2} \cos \varphi_{2}+l_{4}^{\prime} \cos \left(\varphi_{4}-\alpha_{4}\right)-l_{3} \cos \varphi_{3}=x_{O^{\prime}}-x_{C_{0}} \\
l_{2} \sin \varphi_{2}+l_{4}^{\prime} \sin \left(\varphi_{4}-\alpha_{4}\right)-l_{3} \sin \varphi_{3}=y_{0}-l_{1}-s_{1} \\
l_{3} \cos \varphi_{3}-l_{4} \cos \varphi_{4}+s_{5} \sin \alpha=e \cdot \cos \alpha \\
l_{3} \sin \varphi_{3}-l_{4} \sin \varphi_{4}-s_{5} \cos \alpha=e \cdot \sin \alpha .
\end{array}\right.
$$

In the particular case when the articulations B, C and D are collinear, the angle $\alpha_{4}$ is null $\left(\alpha_{4}=0\right)$, so that the scaling equations (8) become:

$$
\left\{\begin{array}{c}
l_{2} \cos \varphi_{2}+l_{4}^{\prime} \cos \varphi_{4}-l_{3} \cos \varphi_{3}=x_{O^{\prime}}-x_{C_{0}} \\
l_{2} \sin \varphi_{2}+l_{4}^{\prime} \sin \varphi_{4}-l_{3} \sin \varphi_{3}=y_{0}-l_{1}-s_{1} \\
l_{3} \cos \varphi_{3}-l_{4} \cos \varphi_{4}+s_{5} \sin \alpha=e \cdot \cos \alpha \\
l_{3} \sin \varphi_{3}-l_{4} \sin \varphi_{4}-s_{5} \cos \alpha=e \cdot \sin \alpha
\end{array}\right.
$$

To solve the system consisting of four partially non-linear scaling equations (9) with 4 unknown variables, we can apply the linearization method $[1,4]$. Thus, in the initial position of the mechanism $\left(s_{1}=0\right)$ we measure (on the scaled kinematic diagram) the angles $\varphi_{2}^{0}, \varphi_{3}^{0}, \varphi_{4}^{0}$ and the linear displacement $s_{5}^{0}$.

In a position of the plane mechanism (from the vicinity of the initial position) for $s_{1}$ imposed, the four variables become: $\varphi_{2}^{0}+\Delta \varphi_{2}, \varphi_{3}^{0}+\Delta \varphi_{3}, \varphi_{4}^{0}+\Delta \varphi_{4}$ and $s_{5}^{0}+\Delta s_{5}$. 
The equations (9) are written:

$$
\left\{\begin{array}{c}
l_{2} \cos \left(\varphi_{2}^{0}+\Delta \varphi_{2}\right)+l_{4}^{\prime} \cos \left(\varphi_{4}^{0}+\Delta \varphi_{4}\right)-l_{3} \cos \left(\varphi_{3}^{0}+\Delta \varphi_{3}\right)=x_{O^{\prime}}-x_{C_{0}} \\
l_{2} \sin \left(\varphi_{2}^{0}+\Delta \varphi_{2}\right)+l_{4}^{\prime} \sin \left(\varphi_{4}^{0}+\Delta \varphi_{4}\right)-l_{3} \sin \left(\varphi_{3}^{0}+\Delta \varphi_{3}\right)=y_{0}-l_{1}-s_{1} \\
l_{3} \cos \left(\varphi_{3}^{0}+\Delta \varphi_{3}\right)-l_{4} \cos \left(\varphi_{4}^{0}+\Delta \varphi_{4}\right)+\left(s_{5}^{0}+\Delta s_{5}\right) \sin \alpha=e \cdot \cos \alpha \\
l_{3} \sin \left(\varphi_{3}^{0}+\Delta \varphi_{3}\right)-l_{4} \sin \left(\varphi_{4}^{0}+\Delta \varphi_{4}\right)-\left(s_{5}^{0}+\Delta s_{5}\right) \cos \alpha=e \cdot \sin \alpha
\end{array}\right.
$$

The linearization of the (10) equations is done with the formulae:

$$
\cos \left(\varphi_{i}+\Delta \varphi_{i}\right)=\cos \varphi_{i}-\Delta \varphi_{i} \cdot \sin \varphi_{i} ; \sin \left(\varphi_{i}+\Delta \varphi_{i}\right)=\sin \varphi_{i}+\Delta \varphi_{i} \cdot \cos \varphi_{i} \cdot i=2,3,4
$$

The system of non-linear equations becomes a system of linear equations:

$$
\left\{\begin{array}{c}
l_{2} \sin \varphi_{2}^{0} \cdot \Delta \varphi_{2}+l_{4}^{\prime} \sin \varphi_{4}^{0} \cdot \Delta \varphi_{4}+l_{3} \cos \varphi_{3}^{0} \cdot \Delta \varphi_{3}=l_{2} \cos \varphi_{2}^{0}+l_{4}^{\prime} \cos \varphi_{4}^{0}+l_{3} \cos \varphi_{3}^{0}-x_{O^{\prime}}+x_{C_{0}} ; \\
l_{2} \cos \varphi_{2}^{0} \cdot \Delta \varphi_{2}+l_{4}^{\prime} \cos \varphi_{4}^{0} \cdot \Delta \varphi_{4}-l_{3} \cos \varphi_{3}^{0} \cdot \Delta \varphi_{3}=y_{0}-l_{1}-s_{1}-l_{2} \sin \varphi_{2}^{0}-l_{4}^{\prime} \sin \varphi_{4}^{0}+l_{3} \sin \varphi_{3}^{0} ; \\
l_{3} \sin \varphi_{3}^{0} \cdot \Delta \varphi_{3}-l_{4} \sin \varphi_{4}^{0} \cdot \Delta \varphi_{4}+\sin \alpha \cdot \Delta s_{5}=l_{3} \cos \varphi_{3}^{0}-l_{4} \cos \varphi_{4}^{0}+s_{5}^{0} \cdot \sin \alpha+e \cdot \cos \alpha ; \\
l_{3} \cos \varphi_{3}^{0} \cdot \Delta \varphi_{3}-l_{4} \cos \varphi_{4}^{0} \cdot \Delta \varphi_{4}-\cos \alpha \cdot \Delta s_{5}=e \cdot \sin \alpha-l_{3} \sin \varphi_{3}^{0}+l_{4} \sin \varphi_{4}^{0}+s_{5}^{0} \cdot \cos \alpha .
\end{array}\right.
$$

This system of scaling equations shall be explicitly written:

$$
\left\{\begin{array}{l}
a_{11} X_{1}+a_{12} X_{2}+a_{13} X_{3}+a_{14} X_{4}=b_{1} \\
a_{21} X_{1}+a_{22} X_{2}+a_{23} X_{3}+a_{24} X_{4}=b_{2} \\
a_{31} X_{1}+a_{32} X_{2}+a_{33} X_{3}+a_{34} X_{4}=b_{3} \\
a_{41} X_{1}+a_{42} X_{2}+a_{43} X_{3}+a_{44} X_{4}=b_{4}
\end{array}\right.
$$

The following notations were used in this system of equations:

$$
X_{1}=\Delta \varphi_{2} ; X_{2}=\Delta \varphi_{3} ; X_{3}=\Delta \varphi_{4} ; X_{4}=\Delta s_{5} .
$$

\section{THE KINEMATIC CALCULATION OF THE PLANAR TRIADIC CHAIN MECHANISM}

We begin from the final stage of the geometrical calculation of the plane triadic chain mechanism, when we know the angular or linear positions of the component elements.

To determine the angular and linear velocities of the kinematic elements in the structure of the mechanism, we consider the previously determined position scaling equations (9).

Each scaling equation is differentiated with respect to time, resulting in the linear system:

$$
\left\{\begin{array}{c}
-l_{2} \sin \varphi_{2} \cdot \dot{\varphi}_{2}-l_{4}^{\prime} \sin \varphi_{4} \cdot \dot{\varphi}_{4}+l_{3} \sin \varphi_{3} \cdot \dot{\varphi}_{3}=0 \\
l_{2} \cos \varphi_{2} \cdot \dot{\varphi}_{2}+l_{4}^{\prime} \cos \varphi_{4} \cdot \dot{\varphi}_{4}-l_{3} \cos \varphi_{3} \cdot \dot{\varphi}_{3}=-\dot{s}_{1} \\
-l_{3} \sin \varphi_{3} \cdot \dot{\varphi}_{3}+l_{4} \sin \varphi_{4} \cdot \dot{\varphi}_{4}+\dot{s}_{5} \sin \alpha=0 \\
l_{3} \cos \varphi_{3} \cdot \dot{\varphi}_{3}-l_{4} \cos \varphi_{4} \cdot \dot{\varphi}_{4}+\dot{s}_{5} \cos \alpha=0
\end{array}\right.
$$

We know the linear velocity of piston $1\left(\dot{s}_{1}\right)$, and the unknown variables in the equations forming this system are the angular velocities $\dot{\varphi}_{2}=\omega_{2}=x_{1}, \dot{\varphi}_{3}=\omega_{3}=x_{2}, \dot{\varphi}_{4}=\omega_{4}=x_{3}$, the linear velocity $\dot{s}_{5}=v_{5}=x_{4}$.

The linear equations system (14) shall be generally written: 


$$
\left\{\begin{array}{l}
a_{11} x_{1}+a_{12} x_{2}+a_{13} x_{3}+a_{14} x_{4}=b_{1} \\
a_{21} x_{1}+a_{22} x_{2}+a_{23} x_{3}+a_{24} x_{4}=b_{2} \\
a_{31} x_{1}+a_{32} x_{2}+a_{33} x_{3}+a_{33} x_{4}=b_{3} \\
a_{41} x_{1}+a_{42} x_{2}+a_{43} x_{3}+a_{44} x_{4}=b_{4}
\end{array}\right.
$$

The angular / linear accelerations are obtained by differentiating with respect to time the velocity scaling equations (14), resulting in:

$$
\left\{\begin{array}{c}
l_{2} \cos \varphi_{2} \cdot \dot{\varphi}_{2}^{2}+l_{2} \sin \varphi_{2} \cdot \ddot{\varphi}_{2}+l_{4}^{\prime} \cos \varphi_{4} \cdot \dot{\varphi}_{4}^{2}+l_{4}^{\prime} \sin \varphi_{4} \cdot \ddot{\varphi}_{4}-l_{3} \cos \varphi_{3} \cdot \dot{\varphi}_{3}^{2}-l_{3} \sin \varphi_{3} \cdot \ddot{\varphi}_{3}=0 ; \\
l_{2} \sin \varphi_{2} \cdot \dot{\varphi}_{2}^{2}-l_{2} \cos \varphi_{2} \cdot \ddot{\varphi}_{2}+l_{4}^{\prime} \sin \varphi_{4} \cdot \dot{\varphi}_{4}^{2}-l_{4}^{\prime} \cos \varphi_{4} \cdot \ddot{\varphi}_{4}-l_{3} \sin \varphi_{3} \cdot \dot{\varphi}_{3}^{2}+l_{3} \cos \varphi_{3} \cdot \ddot{\varphi}_{3}=\ddot{s}_{1} ; \\
\quad-l_{3} \cos \varphi_{3} \cdot \dot{\varphi}_{3}^{2}-l_{3} \sin \varphi_{3} \cdot \ddot{\varphi}_{3}+l_{4} \cos \varphi_{4} \cdot \dot{\varphi}_{4}^{2}+l_{4} \sin \varphi_{4} \cdot \ddot{\varphi}_{4}+\ddot{s}_{5} \sin \alpha=0 ; \\
-l_{3} \sin \varphi_{3} \cdot \dot{\varphi}_{3}^{2}+l_{3} \cos \varphi_{3} \cdot \ddot{\varphi}_{3}+l_{4} \sin \varphi_{4} \cdot \dot{\varphi}_{4}^{2}-l_{4} \cos \varphi_{4} \cdot \ddot{\varphi}_{4}+\ddot{s}_{5} \cos \alpha=0 .
\end{array}\right.
$$

Keeping to the left of the above equations the terms containing accelerations, the system of linear equations (16) shall be written:

$$
\left\{\begin{array}{c}
l_{2} \sin \varphi_{2} \cdot \ddot{\varphi}_{2}+l_{4}^{\prime} \sin \varphi_{4} \cdot \ddot{\varphi}_{4}-l_{3} \sin \varphi_{3} \cdot \ddot{\varphi}_{3}=-l_{2} \cos \varphi_{2} \cdot \dot{\varphi}_{2}^{2}-l_{4}^{\prime} \cos \varphi_{4} \cdot \dot{\varphi}_{4}^{2}+l_{3} \cos \varphi_{3} \cdot \dot{\varphi}_{3}^{2} ; \\
-l_{2} \cos \varphi_{2} \cdot \ddot{\varphi}_{2}-l_{4}^{\prime} \cos \varphi_{4} \cdot \ddot{\varphi}_{4}+l_{3} \cos \varphi_{3} \cdot \ddot{\varphi}_{3}=\ddot{s}_{1}-l_{2} \sin \varphi_{2} \cdot \dot{\varphi}_{2}^{2}-l_{4}^{\prime} \sin \varphi_{4} \cdot \dot{\varphi}_{4}^{2}+l_{3} \sin \varphi_{3} \cdot \dot{\varphi}_{3}^{2} ; \\
-l_{3} \sin \varphi_{3} \cdot \ddot{\varphi}_{3}+l_{4} \sin \varphi_{4} \cdot \ddot{\varphi}_{4}+\ddot{s}_{5} \sin \alpha=l_{3} \cos \varphi_{3} \cdot \dot{\varphi}_{3}^{2}-l_{4} \cos \varphi_{4} \cdot \dot{\varphi}_{4}^{2} ; \\
l_{3} \cos \varphi_{3} \cdot \ddot{\varphi}_{3}-l_{4} \cos \varphi_{4} \cdot \ddot{\varphi}_{4}+\ddot{s}_{5} \cos \alpha=l_{3} \sin \varphi_{3} \cdot \dot{\varphi}_{3}^{2}-l_{4} \sin \varphi_{4} \cdot \dot{\varphi}_{4}^{2}
\end{array}\right.
$$

Solving the system of 4 linear equations (17) the 4 accelerations shall be inferred as unknown variables:

$$
\ddot{\varphi}_{2}=\varepsilon_{2} ; \ddot{\varphi}_{3}=\varepsilon_{3} ; \ddot{\varphi}_{4}=\varepsilon_{4} ; \ddot{s}_{5}=a_{5} .
$$

\section{THE GEOMETRICAL CALCULATION OF THE NEW TRIADIC CHAIN MECHANISM}

We consider the kinematic scheme of the $\mathrm{V}$ mechanism (Figure 5.a) where two symmetrical triadic chains are identified $\operatorname{LT}(2,3,4,5)$ and $\operatorname{LT}(\overline{2}, \overline{3}, \overline{4}, \overline{5})$.
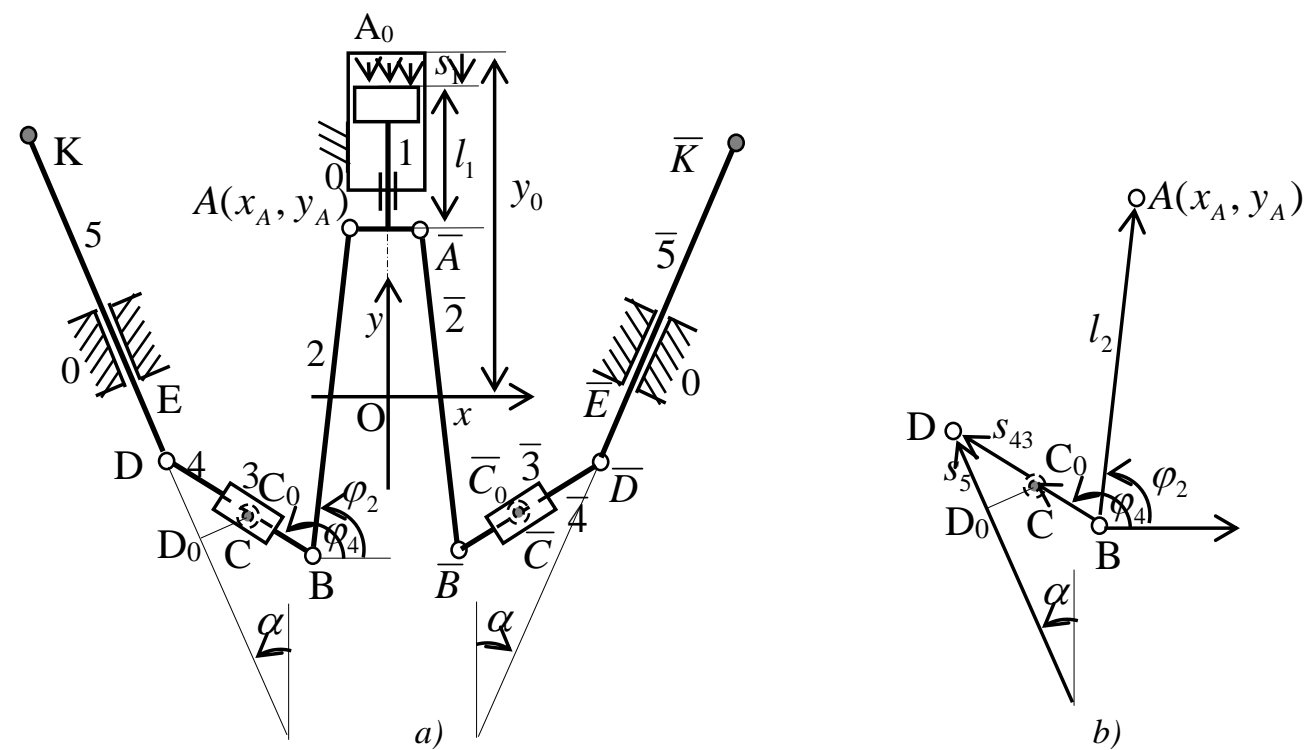

Fig. 5. Kinematic scheme of the new V mechanism $(a)$ and vector contour lines $(b)$. 
Depending on the $y_{0}$ coordinate, the point $A_{0}$, the displacement $s_{1}$ of piston 1 and the lengths $l_{1}$ and $l_{1}^{\prime}=0,5 A \bar{A}$ we determine the coordinates of the articulations $A$ and $\bar{A}$ (Figure 5.b):

$$
x_{A}=-l_{1}^{\prime} ; y_{A}=y_{0}-l_{1}-s_{1} ; x_{\bar{A}}=l_{1}^{\prime} ; y_{\bar{A}}=y_{0}-l_{1}-s_{1} \text {. }
$$

Also, the Cartesian coordinates of the fixed articulations $C_{0}$ and $\bar{C}_{0}$ are known: $x_{C_{0}}=-x_{\bar{C}_{0}} ; y_{C_{0}}=y_{\bar{C}_{0}}$.

The two fixed guides of the bars 5 and $\overline{5}$ (Figure 5.a) are inclined at an $\alpha$ angle as to the axis of the coordinates.

Following only the left kinematic chain, the fixed point $C_{0}$ is situated as to the guide $\Delta_{5}$ at the distance $C_{0} D_{0}=e$, and displacement of the point $D$ as to $D_{0}$ is $D_{0} D=s_{5}$.

The constant lengths of the bars 2 and 4 are known: $A B=l_{2} ; D B=l_{4}$.

We noted as $C$ the mobile point on the bar 4, whose position as to the articulation $D$ was noted as $C D=s_{43}$ and symbolises the relative displacement between elements 4 and 3 .

To determine the instantaneous positions of the proposed mechanism (Figure 5.a), we identify two independent closed contour lines containing the elements $0,2,4,3,0$ and $0,3,4,5,0$.

Using the method of the associated vector contour [1,4], we obtain the following scaling equations:

$$
\begin{aligned}
& l_{2} \cos \varphi_{2}-l_{4} \cos \varphi_{4}+s_{43} \cos \varphi_{4}=x_{A}-x_{C_{0}} \\
& l_{2} \sin \varphi_{2}-l_{4} \sin \varphi_{4}+s_{43} \sin \varphi_{4}=y_{A}-y_{C_{0}} \\
& s_{5} \sin \alpha-s_{43} \cos \varphi_{4}=e \cdot \cos \alpha \\
& s_{5} \cos \alpha-s_{43} \sin \varphi_{4}=e \cdot \sin \alpha .
\end{aligned}
$$

It should be noticed that the first two scaling equations are non-linear having the angular unknown variables $\varphi_{2}$ and $\varphi_{4}$, while the last two equations are partially linear having the unknown variables $s_{43}$ and $s_{5}=s_{50}$.

\section{VELOCITIES AND ACCELERATIONS FOR THE NEW TRIADIC CHAIN V MECHANISM}

Depending on the instantaneous known position of the analysed mechanism (Figure 6.a), we can determine the angular and linear velocities of the led kinematic elements forming the triadic chains $L T(2,3,4,5)$ and $L T(\overline{2}, \overline{3}, \overline{4}, \overline{5})$ of the $4 \mathrm{R}+2 \mathrm{~T}$ type.

We consider the positions/ displacements scaling equations (18) that are differentiated with respect to time, resulting in the velocities equations:

$$
\begin{aligned}
& -l_{2} \sin \varphi_{2} \cdot \dot{\varphi}_{2}+\left(l_{4}-s_{43}\right) \sin \varphi_{4} \cdot \dot{\varphi}_{4}+\cos \varphi_{4} \cdot \dot{s}_{43}=\dot{x}_{A} ; \\
& l_{2} \cos \varphi_{2} \cdot \dot{\varphi}_{2}-\left(l_{4}-s_{43}\right) \cos \varphi_{4} \cdot \dot{\varphi}_{4}+\sin \varphi_{4} \cdot \dot{s}_{43}=\dot{y}_{A} ; \\
& s_{43} \sin \varphi_{4} \cdot \dot{\varphi}_{4}-\cos \varphi_{4} \cdot \dot{s}_{43}+\sin \alpha \cdot \dot{s}_{5}=0 ; \\
& s_{43} \cos \varphi_{4} \cdot \dot{\varphi}_{4}+\sin \varphi_{4} \cdot \dot{s}_{43}-\cos \alpha \cdot \dot{s}_{5}=0 .
\end{aligned}
$$

The obtained equations (19) are linear equations forming a linear system that leads to the further calculation of the angular / linear velocities $\dot{\varphi}_{2}=\omega_{2}, \dot{\varphi}_{4}=\omega_{4}, \dot{s}_{43}=v_{43}, \dot{s}_{5}=v_{5}$. 
The angular $\left(\varphi_{2}, \varphi_{4}\right)$ and linear $\left(s_{43}, s_{5}\right)$ positions of the elements of the triadic chain are determined in the previous stage. The components of the point A velocity are: $\dot{x}_{A}=0 ; \dot{y}_{A}=-v_{1}$.

Generally, the velocity scaling equations are written:

$$
\left\{\begin{array}{c}
a_{11} x_{1}+a_{12} x_{2}+a_{13} x_{3}+a_{14} x_{4}=0 \\
a_{21} x_{1}+a_{22} x_{2}+a_{23} x_{3}+a_{24} x_{4}=-v_{1} \\
a_{31} x_{1}+a_{32} x_{2}+a_{33} x_{3}+a_{34} x_{4}=0 \\
a_{41} x_{1}+a_{42} x_{2}+a_{43} x_{3}+a_{44} x_{4}=0
\end{array}\right.
$$

The following notations were used in the system of linear scaling equations (20):

- for variable or constant coefficients: $a_{11}=-l_{2} \sin \varphi_{2} ; a_{12}=\left(l_{4}-s_{43}\right) \sin \varphi_{4} ; a_{13}=\cos \varphi_{4} ; a_{14}=0$;

$$
\begin{aligned}
& a_{21}=l_{2} \cos \varphi_{2} ; a_{22}=-\left(l_{4}-s_{43}\right) \cos \varphi_{4} ; a_{23}=\sin \varphi_{4} ; a_{24}=0 ; \\
& a_{31}=0 ; a_{32}=s_{43} \sin \varphi_{4} ; a_{33}=-\cos \varphi_{4} ; a_{34}=\sin \alpha ; a_{41}=0 ; a_{42}=s_{43} \cos \varphi_{4} ; a_{43}=\sin \varphi_{4} ; a_{44}=-\cos \alpha .
\end{aligned}
$$

- for unknown variables / angular or linear velocities: $x_{1}=\dot{\varphi}_{2} ; x_{2}=\dot{\varphi}_{4} ; x_{3}=\dot{s}_{43} ; x_{5}=\dot{s}_{5}$.

In the third stage, knowing the angular / linear velocities of the kinematic elements 2, 3, 4 and 5 from the triadic chain, we determine the angular / linear accelerations of these kinematic elements. Thus, the explicit scaling equations (19) are differentiated with respect to time and the following are obtained:

$$
\begin{gathered}
-l_{2} \sin \varphi_{2} \cdot \ddot{\varphi}_{2}+\left(l_{4}-s_{43}\right) \sin \varphi_{4} \cdot \ddot{\varphi}_{4}+\cos \varphi_{4} \cdot \ddot{s}_{43}=l_{2} \cos \varphi_{2} \cdot \dot{\varphi}_{2}^{2}-l_{4} \cos \varphi_{4} \cdot \dot{\varphi}_{4}^{2}+2 \sin \varphi_{4} \cdot \dot{s}_{43} \cdot \dot{\varphi}_{4} \\
l_{2} \cos \varphi_{2} \cdot \ddot{\varphi}_{2}-\left(l_{4}-s_{43}\right) \cos \varphi_{4} \cdot \ddot{\varphi}_{4}+\sin \varphi_{4} \cdot \ddot{s}_{43}=l_{2} \sin \varphi_{2} \cdot \dot{\varphi}_{2}^{2}-l_{4} \sin \varphi_{4} \cdot \dot{\varphi}_{4}^{2}-2 \cos \varphi_{4} \cdot \dot{s}_{43} \cdot \dot{\varphi}_{4} ; \\
s_{43} \sin \varphi_{4} \cdot \ddot{\varphi}_{4}-\cos \varphi_{4} \cdot \ddot{s}_{43}+\sin \alpha \cdot \ddot{s}_{5}=-s_{43} \cos \varphi_{4} \cdot \dot{\varphi}_{4}^{2}-2 \sin \varphi_{4} \cdot \dot{s}_{43} \cdot \dot{\varphi}_{4} \\
s_{43} \cos \varphi_{4} \cdot \ddot{\varphi}_{4}-\sin \varphi_{4} \cdot \ddot{s}_{43}+\cos \alpha \cdot \ddot{s}_{5}=-s_{43} \sin \varphi_{4} \cdot \dot{\varphi}_{4}^{2}-2 \cos \varphi_{4} \cdot \dot{s}_{43} \cdot \dot{\varphi}_{4}
\end{gathered}
$$

From this system of linear equations we determine the accelerations: $\ddot{\varphi}_{2}, \ddot{\varphi}_{4}, \ddot{s}_{43}, \ddot{s}_{5}$.

\section{CONCLUSIONS}

Starting from the current dyadic chain kinematic scheme of the $\mathrm{V}$ high voltage power switcher, the paper suggests new kinematic diagrams with a triadic structure. For the first kinematic scheme proposed, based on a triadic chain of the $5 \mathrm{R}+\mathrm{T}$ type, we have analysed two versions having a different geometrical structure.

The second kinematic scheme is based on a triadic chain of the $4 \mathrm{R}+2 \mathrm{~T}$ type, having the possibility to optimize the operation of the $\mathrm{V}$ mechanism. For each new kinematic scheme the geometrical and kinematic analysis method has been developed, presenting all the stages of the analytic calculations of displacements, velocities and accelerations, especially for the translating bar with the power switch point.

\section{REFERENCES}

[1] Antonescu, P., Antonescu, O., Mechanisms and the dynamics of machines, Ed. Printech, Bucharest, 2005.

[2] Nedela, N., Antonescu, O., Mechanisms used as medium voltage electric power switchers, Journal of Mechanisms and Manipulators, vol. 8, no 1, 2009.

[3] Nedela, N., Geonea, I., Mechanisms used for high voltage switch devices, Journal of Mechanisms and Manipulators, vol. 9, no. 1, 2010, p. 51-58.

[4]. Nedela, N., A Geometrical analysis and synthesis of mechanisms in the electro technical field, Doctoral thesis, Politehnica University of Bucharest, 2012. 